\title{
Article
}

\section{The influence of experience upon information-sampling and decision- making behaviour during risk assessment in military personnel}

Godwin, H.J., Liversedge, Simon Paul, Kirkby, Julie A., Boardman, M., Cornes, K. and Donnelly, Nick

Available at http://clok.uclan.ac.uk/22362/

Godwin, H.J., Liversedge, Simon Paul ORCID: 0000-0002-8579-8546, Kirkby, Julie A., Boardman, M., Cornes, K. and Donnelly, Nick (2015) The influence of experience upon information-sampling and decision-making behaviour during risk assessment in military personnel. Visual Cognition, 23 (4). pp. 415-431. ISSN 1350-6285

It is advisable to refer to the publisher's version if you intend to cite from the work. http://dx.doi.org/10.1080/13506285.2015.1030488

For more information about UCLan's research in this area go to http://www.uclan.ac.uk/researchgroups/ and search for <name of research Group>.

For information about Research generally at UCLan please go to http://www.uclan.ac.uk/research/

All outputs in CLoK are protected by Intellectual Property Rights law, including Copyright law. Copyright, IPR and Moral Rights for the works on this site are retained by the individual authors and/or other copyright owners. Terms and conditions for use of this material are defined in the policies page. 
Running head: RISK ASSESSMENT BEHAVIOUR

The Influence of Experience upon Information-sampling and Decision-Making Behaviour during Risk Assessment in Military Personnel

Hayward J. Godwin ${ }^{1}$, Simon P. Liversedge ${ }^{1}$, Julie A. Kirkby ${ }^{2}$, Michael Boardman ${ }^{3}$, Katherine

\author{
Cornes $^{3}$, Nick Donnelly ${ }^{1}$ \\ ${ }^{1}$ University of Southampton, UK \\ ${ }^{2}$ Bournemouth University, UK \\ ${ }^{3}$ Defence Science and Technology Laboratory
}

Author Note

Hayward J. Godwin, Simon P. Liversedge, and Nick Donnelly, School of Psychology, University of Southampton, UK. Julie A. Kirkby, Bournemouth University, UK. Michael Boardman and Katherine Cornes, Defence Science and Technology Laboratory, UK.

Correspondence regarding this article should be addressed to Hayward J. Godwin, University of Southampton, School of Psychology, Highfield, Southampton, Hampshire, SO17 1BJ. Tel: +44(0)2380 595078; Email: hayward.godwin@soton.ac.uk. 


\begin{abstract}
We examined the influence of experience upon information-sampling and decisionmaking behaviour in a group of military personnel as they conducted risk assessments of scenes photographed from patrol routes during the recent conflict in Afghanistan. Their risk assessment was based on an evaluation of Potential Risk Indicators during examination of each scene. We found that both participant groups were equally likely to fixate Potential Risk Indicators, demonstrating similarity in the selectivity of their information-sampling. However, the inexperienced participants made more revisits to Potential Risk Indicators, had longer response times, and were more likely to decide that the scenes contained a high level of risk. Together, these results suggest that experience primarily modulates decision-making behaviour. We discuss potential routes to train personnel to conduct risk assessments in a more similar manner to experienced participants.
\end{abstract}

Acknowledgements: This research was supported by funding from the Defence Science and Technology Laboratory. Content includes material subject to Dstl @ C Crown copyright (2015). Licensed under the terms of the Open Government Licence except where otherwise stated. To view this licence, visit http://www.nationalarchives.gov.uk/doc/open-governmentlicence/version/3 or write to the Information Policy Team, The National Archives, Kew, London TW9 4DU, or email: psi@ nationalarchives.gsi.gov.uk.

Keywords: experience, expertise, decision-making, eye movements 
Imagine that you are a soldier on patrol along a route during an on-going conflict, such as that depicted in Figure 1. As you proceed along your route, you will need to continuously monitor the environment for a number of potential forms of threat that could emerge or become visible at any moment. For instance, you will need to monitor the path that you are taking for Improvised Explosive Devices (IEDs) buried in the ground. You will also need to monitor the broader environment for locations where an enemy force might choose to launch an attack upon you and your team. Failure to detect or rapidly respond to threats in this type of environment will potentially have very significant consequences. In the present study, we examined how military personnel assess scenes of this type for risks, and focused on the extent that live combat experience modulated their behaviour as they were engaged in a novel task involving risk assessments of scenes photographed during the recent conflict in Afghanistan. We compared the behaviour of two groups of participants: an experienced group, with live combat experience and an inexperienced group, who had received basic training on risk assessment but did not have live combat experience. Note that other studies that have examined the performance of experienced searchers often compare expert versus novice behaviour. In the context of the present study, we refer to our two groups as experienced versus inexperienced since even the inexperienced group had received training, making it inaccurate to refer to them as 'novices'. 


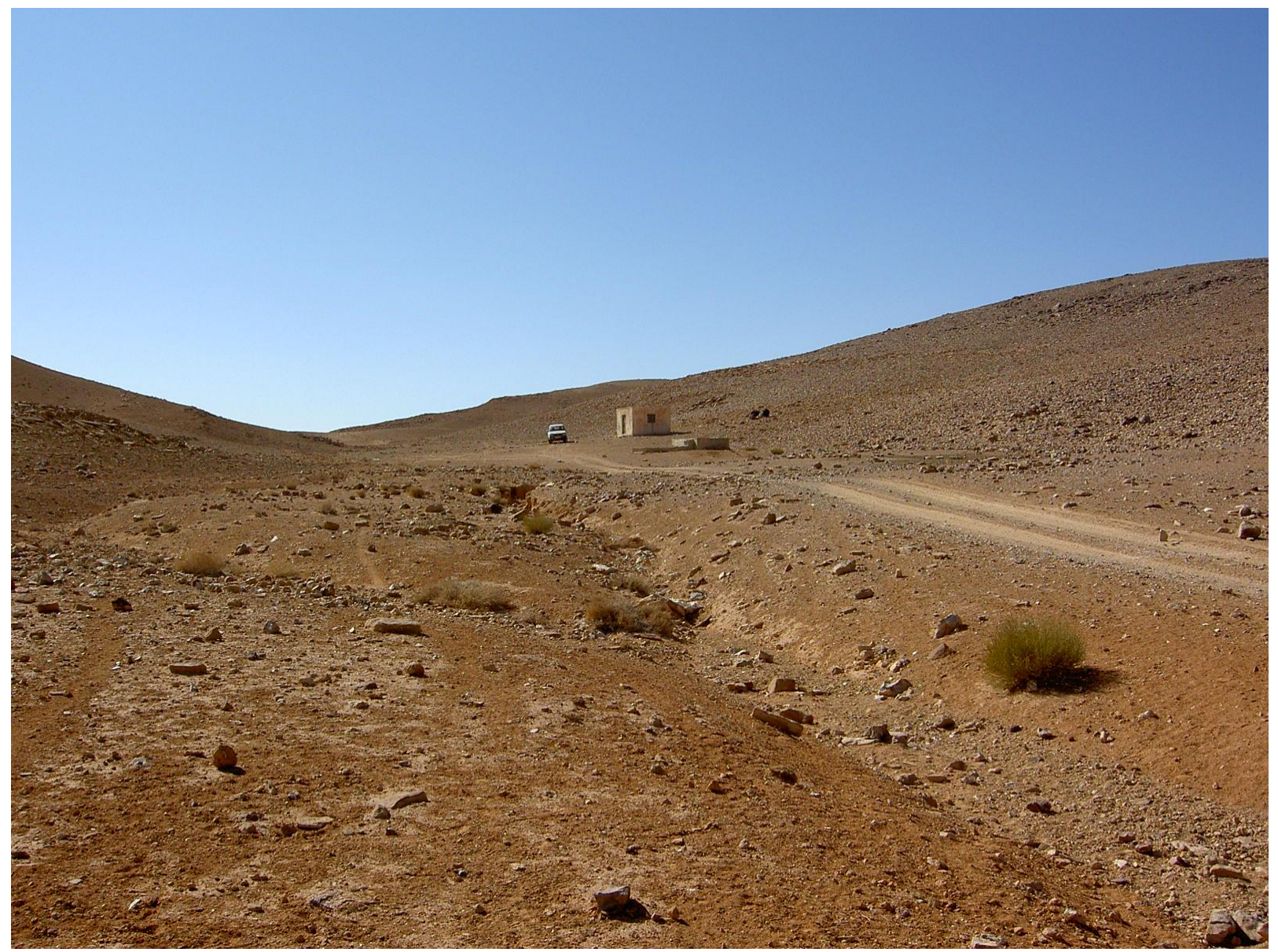

Figure 1. Sample scene of the type used in the study. In this example, observers must examine the ground for signs of disturbance, and check the building and surrounding hillside for locations where an opposing force might choose to launch an attack. Note that this image does not contain any threats/risks.

There exists an extensive body of prior research that has examined the role that experience and expertise plays upon visual cognitive processes (Reingold \& Sheridan, 2011). Typically these studies have found that increasing experience with a given task improves performance substantially. The key issue that arises from this shift in performance is how experience improves performance: is it through improving information-sampling (i.e., the objects 
or areas of a stimuli that are inspected, typically determined using eye-tracking) or decisionmaking (i.e., the resultant decision made regarding whether a target is present or absent from a stimulus)? Both information-sampling and decision-making are vital components in the detection of visual targets. When information-sampling fails to guide the eyes to targets, those targets are often missed (Chapman \& Underwood, 1998; Godwin, Menneer, Riggs, Cave, \& Donnelly, 2015; Kundel \& Nodine, 1975; Nodine \& Mello-Thoms, 2000). When the targets are examined, decision-making can also fail to detect the targets once fixed, causing the targets to again be missed (Cain, Adamo, \& Mitroff, 2013; Godwin et al., 2015; Nodine \& Kundel, 1987; Schwark, MacDonald, Sandry, \& Dolgov, 2013).

The studies described above focus on the various benefits that arise from experience with a given task, both in terms of reduced time spent localising a target (as a result of a benefit to information-sampling behaviour) and consequently a benefit to decision-making behaviour. However, it is not always the case that experience with a given task reduces time spent searching while also increasing response accuracy. Studies of airport X-ray baggage screeners (Biggs, Cain, Clark, Darling, \& Mitroff, 2013; Biggs \& Mitroff, 2014a, 2014b) have shown that experienced searchers are more accurate than inexperienced searchers. However, in X-ray baggage screeners, there is a cost associated with this increase in accuracy: search is more accurate but less rapid for experienced searchers, who approach search in a consistently more cautious manner. This finding suggests that experienced X-ray baggage screeners may be more exhaustive in their searchers (Wolfe, Brunelli, Rubinstein, \& Horowitz, 2013), perhaps due to spending longer examining each object or examining more objects (Godwin, Menneer, Cave, Thaibsyah, \& Donnelly, 2014). 
In visual search tasks, participants typically give a categorical present/absent decision for each trial. Here, we examine information-sampling and decision-making in a task that is very different to those that have been examined before this point, and does not necessarily involve such a clear-cut, categorical decision-making process: risk assessment in high-threat combat environments. As would be expected, it is not possible to engage observers in a live version of this task, so we instead asked them to inspect scenes such as those in Figure 1. We asked participants not to make a categorical yes/no or present/absent decision regarding whether they believed a target to be present, since that is not the task that they are experienced with engaging in while in combat environments. Instead, we asked them to decide whether, if they were on patrol within the scene depicted, they would halt their patrol, or whether they would continue their patrol. This is the decision that, when on patrol, military personnel are required to make continuously. It is more nuanced than simply responding present/absent, since to make such a decision, as we shall now describe, requires the integration and collation of multiple sources of information.

Threats when on patrol in a combat environment can take multiple forms and are, in many senses, subject to interpretation. We identified Potential Risk Indicators (PRIs) with the assistance of a group of expert specialised explosive ordinance disposal operators who had undertaken duties in Afghanistan. The 'potential' aspect of the PRIs is vital in understanding the fact that this task does not involve clear-cut yes/no decisions in relation to threat: they are only potential risks insofar as they could (with some likelihood) serve as a point, object or region of significant risk to the observer, either at the current moment, or in the future.

The PRIs were categorised into two groups: Direct PRIs and Indirect PRIs. Direct PRIs consisted of overt signals (e.g., physical signs of digging or physical disturbance) that indicated 
that an IED had been emplaced within the environment. They also consisted of components of IEDs that may be visible to the observer (e.g., wires). Indirect PRIs consisted of areas in the scene that would be advantageous locations from which to launch an attack onto a patrol (or from where to detonate an IED). The indirect PRIs were a more abstract class of indicator than the direct PRIs. Rather than consisting of a specific physical object or visual cue, as is the case with the direct PRIs, the indirect PRIs required detailed evaluation of configurations of objects in relation to each other, and in relation to the intentions of enemy individuals as to whether they afforded the potential for a strategic enemy advantage.

While on patrol in combat environments there could be multiple PRIs visible to the observer (e.g., footprints, vantage points, etc.). It is the task of the military personnel on patrol to inspect, interpret and decide whether the PRIs that are visible cross some threshold for halting the patrol, taking a different route, or calling for assistance. In some instances, direct components of an IED may be visible and detected, causing a 'halt patrol' response. In other instances, however, the evidence for the presence of an IED may be less direct, and may only arise as a result of there be multiple Indirect PRIs indicating that an IED could be present. Taken together, the risk assessment task is not just to search for IEDs, vantage points, or any other PRI and then respond 'halt patrol': the decision-making involved can go beyond the requirement to make a categorical yes/no or present/absent decision, as in standard visual search tasks, and require a nuanced, balanced decision based upon multiple sources PRIs.

With that in mind, here, we were interested in the participants' risk assessments of the scenes. We sought to better understand the information-sampling and subsequent decisionmaking that took place when observers made careful, nuanced decisions regarding whether or not to continue patrolling through a given scene. We tapped into information-sampling by 
examining participants' eye movement behaviour, since this allows us to precisely measure the nature of the PRIs that are examined prior to a decision, as well as the duration spent examining those PRIs. This, in turn, enabled us to understand the scene characteristics that resulted in haltpatrol versus continue-patrol decisions, as well as the eye-movement behaviour and informationsampling that resulted in halt-patrol versus continue-patrol decisions. Based on previous research, we predicted that the experienced participants would be more rapid in their responses than the inexperienced participants. We also predicted that the experienced participants would show enhanced information-sampling compared to the inexperienced participants, evidenced in terms of spending more time inspecting PRIs. Since it was not possible to measure response accuracy in this task, we divided the analyses in terms of whether participants responded 'haltpatrol' and whether they responded 'continue patrol'. This enabled us to tap in directly to the behaviour that preceded each type of decision, allowing us to gain insights into the formulation of the different decisions. We also examined the likelihood that participants would respond 'haltpatrol' versus 'continue-patrol', focusing on how the scene characteristics influenced the decisions that were made: again, this was in order to better understand the factors that influenced each type of decision. In particular, we explored whether the overall area of the display covered by the different PRI types caused participants to be more likely to respond 'halt-patrol', under the assumption that having more PRIs, and larger PRIs in a scene should make them more likely to report that the route is too dangerous to continue patrolling through. Since there were no correct or incorrect answers, we treated the behaviour of the experienced participants as the 'gold standard' for performance.

\section{Method}




\section{Participants}

Forty-two participants, all of whom were military personnel, were recruited for the study (21 experienced participants, 21 inexperienced participants). All participants had been given training in understanding and interpreting the different forms of direct and indirect PRIs. This training was provided by personnel who had been trained at the Defence Munitions and Search School. Personnel had also received Mission Specific Training where the threats specific to Afghanistan (e.g. the most likely types of IED and the most likely locations for IEDs to be emplaced) had been highlighted. The experienced participants had been given the same basic training and had spent at least 6 months using that training to assess the presence or absence of threats in a live combat environment (Afghanistan).

\section{Apparatus}

Eye movement data were recorded using a SR-Research Eyelink 1000 eye tracker operating at a frequency of $1000 \mathrm{~Hz}$ (i.e., one sample every millisecond). During testing, participants rested their chin on a chin rest and forehead against a head restraint facing a 21 " CRT monitor at a viewing distance of $71 \mathrm{~cm}$. The full display subtended $25^{\circ} \times 32^{\circ}$ of visual angle. All responses were made using an SR-Research button box, and all data were processed and examined using SR-Research's DataViewer software for eye tracking data. A calibration was only accepted when the average error was within $0.5^{\circ}$ of visual angle and the maximum error did not exceed $1^{\circ}$ of visual angle.

\section{Stimuli}


Participants were presented with 50 photographs of scenes taken by military personnel while on patrol in Afghanistan. The images were selected following consultation and interviews with military search specialists who highlighted PRIs in the scenes. Twenty-five of the images were known to contain an IED (we call these Known Threat images), and the remaining images were not known to contain an IED (note again that this did not mean that we were certain that these images did not contain an IED, so we call these Unknown Threat images). When selecting the images, we took steps to match the two image types as much as possible. For each Known Threat image, we selected an Unknown Threat image that was of similar appearance or scene type (e.g., urban, rural, desert, etc.) in order to limit the possibility that there were systematic basic differences in the Known and Unknown Threat images. We compared the images in terms of the total proportion of each displays occupied by each PRI type, and in terms of the number of each PRI type in an image. Direct PRIs occupied a similar proportion of the Known and Unknown Threat images (Known Threat: 0.25, SEM=0.04; Unknown Threat: 0.18, SEM=0.02); Indirect PRIs also occupied a similar proportion of the Known and Unknown Threat images (Known Threat; 0.08, SEM =0.02; Unknown Threat: 0.08, 0.02). There was a similar number of Direct PRIs in the Known and Unknown Threat images (Known Threat: 4.4, SEM=0.3; Unknown Threat: 4.0, SEM=0.2); likewise, there was a similar number Indirect PRIs in the Known and Unknown Threat images (Known Threat; 2.0, SEM =0.3; Unknown Threat: 2.1, 0.2). However, it should be noted that there were more Direct PRIs than Indirect PRIs, and the Direct PRIs also occupied a larger total proportion of the displays than the Indirect PRIs. Given that the scenes were digital photographs of real scenes in Afghanistan, it was not possible to control this aspect of the stimuli, and we recognise a priori, that the effects we report should be assessed in line with this difference. 


\section{Design and Procedure}

The experimental trials were preceded by 5 practice trials (which were not repeated as part of the main study). The experimental trials were presented in a different random order for each participant. Each trial was initiated by the experimenter after a drift correct procedure. Following the drift correct, a black square subtending $1.5^{\circ}$ of visual angle was positioned randomly on the display. A gaze-contingent trigger was set up so that participants had to fixate this square for $100 \mathrm{~ms}$ before the display was presented. The fixation point was employed to prevent participants repeatedly fixating the screen centre at trial onset. Once the stimulus was displayed, participants were required to inspect the scene and were given an unlimited period of time to respond. They were instructed to imagine that they were on patrol in the area pictured. With that in mind, their task was to decide whether they would be content to continue-patrolling through the area shown in the image, or whether they believed a threat to be present in the scene. To this extent, they were required to make a threat evaluation. Participants could make one of two responses using the button box provided: one indicated that they would be willing to continue their patrol (continue-patrol) and the second indicated that they would be unwilling to continue their patrol (halt-patrol). The study had a 2 (Experience: Experienced, Inexperienced) $\mathrm{x}$ 2 (Stimulus Type: Known Threat, Unknown Threat) design.

\section{Results}

We began by examining behavioural decision-making measures to explore threat assessment. We first considered responses made, and the time taken to make a response. We then explored the eye movement data to examine the influence of experience upon eye 
movements during threat evaluations, and to determine whether the PRIs were examined in a similar manner for both inexperienced and experienced participants.

\section{Decision-Making Metrics}

\section{Time Taken to Decide 'Halt-Patrol' and 'Continue-Patrol'}

In order to explore the decision-making of the participants, we examined median RTs using a mixed ANOVA with a 2 (Experience: Experienced, Inexperienced) x 2 (Response: halt, continue) design (see Table 1). This revealed a main effect of Experience $(F(1,40)=7.8, p=$ $.008)$, and of Response $(F(1,40)=12.07, p=.001)$, but no interaction between the two factors $(F$ $<1, p=.68$ ). As can be seen in Table 1, continue-patrol RTs were longer than halt-patrol RTs (mimicking traditional visual search tasks where analogous correct responses on "target-absent" trials have longer RTs than analogous "target-present” trials: see Chun \& Wolfe, 1996), and furthermore RTs for the inexperienced participants were significantly longer than the experienced participants.

\section{Probability of Responding 'Halt-Patrol'}

One of our key goals in the present study was to better understand the factors that influence continue-patrol versus halt-patrol decisions. In order to achieve this, we examined the probability that participants responded 'halt-patrol' on each trial, using a Linear Mixed Model (LME). LMEs offer a high level of statistical power, being based on each observation, rather than the mean of a given dependent variable (Baayen, 2008). Our model included as random factors participant and stimulus (i.e., scene image). Since this was a binomial model, we coded 'halt-patrol' responses with a value of 1 , and 'continue-patrol' responses with a value of 0 . All 
slopes and estimates can therefore be interpreted in terms of increasingly positive values leading to participants being more likely to respond 'halt-patrol'.

We began the model-fitting process by constructing a model with the full set of interactions between the factors. We then iterated through different versions of the model, proceeding until we reached the model that had the optimal level of fit, excluding models that failed to converge. As fixed effects, we included the following factors: the Experience level of the participants (inexperienced, experienced), Direct PRI Area (a continuous variable which was log-transformed and coded the overall area in degrees of visual angle covered by Direct PRIs), Indirect PRI Area (a continuous variable also log-transformed coding the area covered by Indirect PRIs), and finally IED Status (set to either unknown or known). For IED Status, we knew that half of the scenes certainly contained IEDs, these were set as having their IED Status known to be present. For the scenes that we did not know whether they contained IEDs, these were set as having their IED status to be unknown to be present. Note that this is not equivalent, as noted in the Introduction, to having target-present/target-absent trials, since again we simply did not know whether some of the images contained IEDs. Still, we reasoned that, since the IEDs in the unknown group were not overtly visible, participants in both groups should therefore be less likely to respond 'halt-patrol' for the unknown versus the known IED group.

The final model, presented in Table 2, and depicted in Figure 2, comprised a set of main effects only, and included Experience, Direct PRI Area, and IED Status. There were no interactions. As can be seen in Figure 2, which plots the mean probability of responding 'haltpatrol' for each scene, broken down for the two participant groups, this approach yielded some important insights. First, since Experience had a negative coefficient, and since the reference level for this factor was the inexperienced participants, this negative coefficient demonstrates 
that the inexperienced participants were more likely to respond 'halt-patrol' than the experienced participants. Second, since Direct PRI Area had a positive coefficient, this demonstrates that increasing the area of Direct PRIs within the scenes increased the likelihood that participants would respond 'halt-patrol', as expected. Third, IED Status had a positive coefficient, and since the reference level for this factor was for the unknown trials, the positive value indicates that the trials known to contain IEDs were more likely to receive a halt-patrol response than those not known to contain IEDs.

\section{Table 1}

Table of Medians and Means for Behavioural and Global Eye Movement Measures. Parentheses indicate SEM

\begin{tabular}{|c|c|c|c|c|c|}
\hline Experience & Response & $\begin{array}{l}\text { Median } \\
\text { RT (ms) }\end{array}$ & $\begin{array}{l}\text { Mean Fixation } \\
\text { Duration (ms) }\end{array}$ & $\begin{array}{c}\text { Mean } \\
\text { Number of } \\
\text { Fixations }\end{array}$ & $\begin{array}{c}\text { Mean Saccade } \\
\text { Amplitude } \\
\text { (deg/visual angle) }\end{array}$ \\
\hline \multirow[t]{2}{*}{ Experienced } & $\begin{array}{l}\text { Continue- } \\
\text { patrol }\end{array}$ & $\begin{array}{c}13678 \\
(1208)\end{array}$ & $272(7.15)$ & $44.4(3.68)$ & $4.87(0.16)$ \\
\hline & $\begin{array}{l}\text { Halt- } \\
\text { patrol }\end{array}$ & $\begin{array}{l}11685 \\
(1082)\end{array}$ & $283(7.72)$ & $37.1(3.02)$ & $4.69(0.14)$ \\
\hline \multirow[t]{2}{*}{ Inexperienced } & $\begin{array}{c}\text { Continue- } \\
\text { patrol }\end{array}$ & $\begin{array}{l}20968 \\
(2433)\end{array}$ & 267 (5.69) & $67.8(7.98)$ & $4.55(0.16)$ \\
\hline & $\begin{array}{l}\text { Halt- } \\
\text { patrol }\end{array}$ & $\begin{array}{l}18431 \\
(2219)\end{array}$ & $276(6.69)$ & $58.2(6.46)$ & $4.29(0.11)$ \\
\hline
\end{tabular}

Table 2. 
Fixed Effect Estimates and Results for the Linear Mixed Effects Model for the Probability of Responding 'Halt-Patrol' Analyses

\begin{tabular}{lcc}
\hline \multicolumn{1}{c}{ Factor } & Estimate & $z$ \\
& & \\
& & \\
Intercept & $-5.91(2.85)$ & $-2.08^{*}$ \\
Experience (inexperienced, experienced) & $-0.66(0.25)$ & $-2.68^{* *}$ \\
Direct PRI Area & $0.57(0.25)$ & $2.33^{*}$ \\
Indirect PRI Area & - & - \\
IED Status (unkown, known) & $0.71(0.35)$ & $2.06^{*}$ \\
\end{tabular}

Note. $\mathrm{z}$ values \pm 1.96 are treated as 'significant'. Parentheses indicate SEM. 


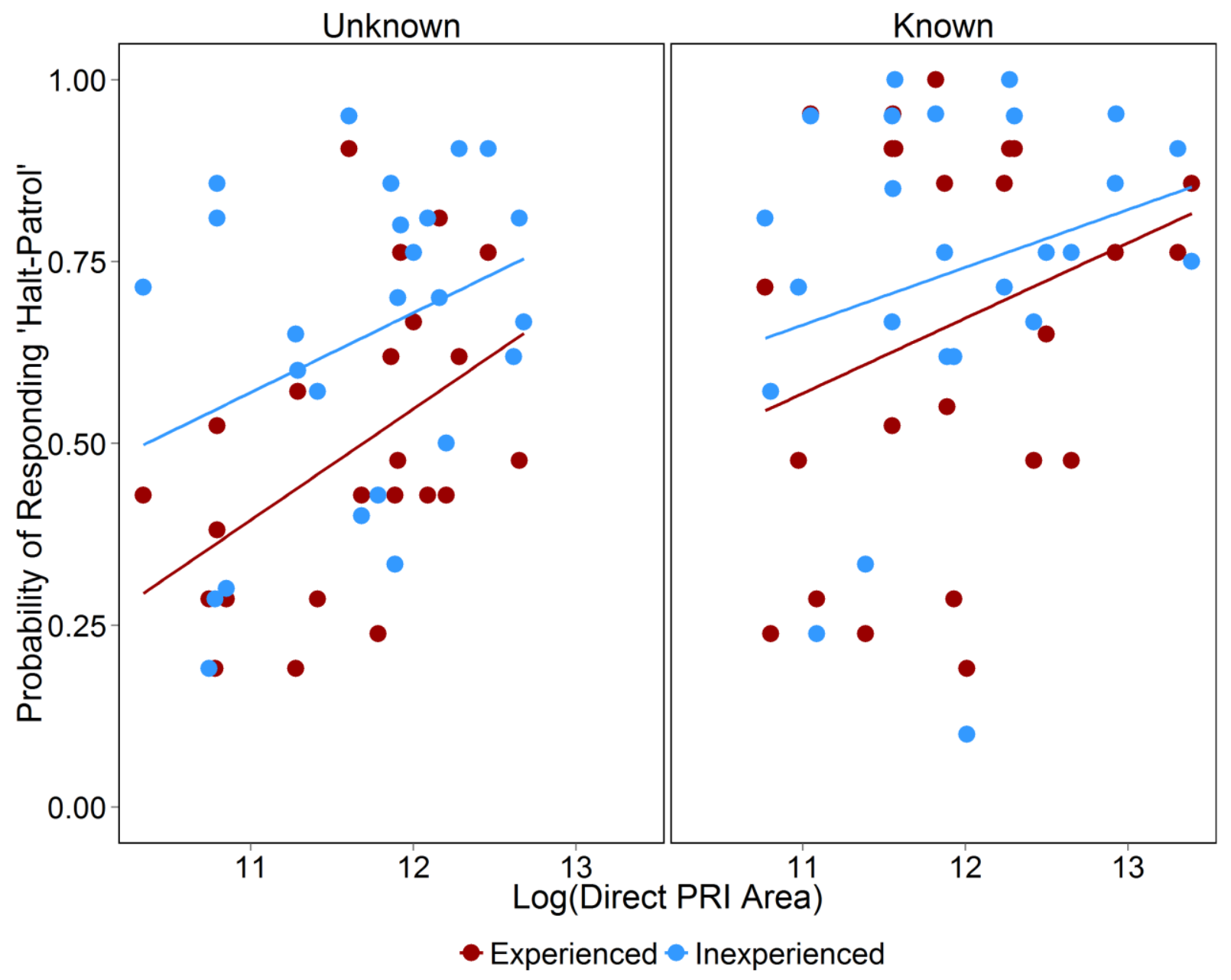

Figure 2. Probability of Responding 'Halt-Patrol' as a function of Experience, IED Status and Direct PRI Area. Each point represents the mean probability of responding 'halt-patrol' and $\log ($ Direct PRI Area) for a given scene. The values have been broken down for each factor, though the factors did not interact within the LME. The break-down has been provided for visualisation purposes only.

\section{Eye Movement Data Processing}

All fixations of less than $60 \mathrm{~ms}$ or greater than $1200 \mathrm{~ms}$ were removed from the dataset. This resulted in the removal of $1.2 \%$ of all fixations, leaving a final dataset of approximately 105,000 fixations.

\section{Global Eye Movement Measures}


Finding a difference in RTs for the experienced versus inexperienced participants raises an interesting question in terms of their search behaviour. Was it the case that the inexperienced participants had longer RTs because their fixation durations were longer compared to experienced participants, indicating a qualitative difference in information processing, or was it the case that there were no differences in fixation durations, but a difference in the number of fixations made by the two participant groups? To address this question, we examined global eye movement measures (mean fixation duration, mean number of fixations) using a series of mixed 2 (Experience: Experienced, Inexperienced) x 2 (Response: Halt-patrol, Continue-patrol) ANOVAs (see Table 1). We also examined the saccade amplitude (in degrees) in order to determine whether there were any systematic differences in the extent to which experienced and inexperienced participants explored the scene.

For the fixation durations, there was evidence of increased fixation durations prior to a halt-patrol response than a continue-patrol response $(F(1,40)=31.16, p<.0001)$, with no other effects reaching significance $(F \mathrm{~s}<1)$. For the fixation counts, there was a main effect of Experience $(F(1,40)=8.12, p=.007)$, with fewer fixations made by the experienced than the inexperienced participants, and of Response $(F(1,40)=22.36, p<.001)$, with a greater number of fixations made prior to continue-patrol responses than halt-patrol responses. There was, however, no interaction between the two factors $(F<1, p=.5)$. Finally, saccade amplitudes were greater prior to halt-patrol than continue-patrol responses $(F(1,40)=13.66, p=.001)$, though no other effects or interactions reached significant $\left(F_{\mathrm{s}}<3.4, p \mathrm{~s}>.075\right)$.

To summarise, these results indicate that the duration of fixations in experienced and inexperienced participants were similar, though the number of fixations made was reduced for experienced than inexperienced participants. Also, participants made more fixations and had 
longer saccade amplitudes prior to continue-patrol responses. This latter result is analogous to target-absent responses being longer than target-present responses in standard visual search experiments (Chun \& Wolfe, 1996).

\section{Potential Risk Indicator Analyses}

The global measures indicate that the increase in RTs for inexperienced participants was due to an increase in the number of fixations made. This, in turn, raises a further question: were the inexperienced participants making a greater number of fixations on the same aspects of the scene when assessing risk as the experienced participants, or instead, were they making additional fixations on different regions of the scene than those fixated by the experienced participants? To address this question, we examined eye movement behaviour in relation to the two PRI types. This also enabled us to determine whether both participant groups searched for both types of PRI to a similar degree as they formulated their assessments of risk. We began by examining the proportion of trial time spent fixating the different PRIs (to counteract the substantial differences in total trial duration between the experienced and inexperienced participants) using a series of mixed 2 (Experience: Experienced, Inexperienced) x 2 (PRI Type: Direct, Indirect) x 2 (Response: Halt-patrol, Continue-patrol) ANOVAs (see Table 3). These analyses revealed an effect of PRI Type $(F(1,40)=1182.3, p<.0001)$, of Response $(F(1,40)=36.29, p<.0001)$, and an interaction between the two factors $(F(1,40)=20.39, p<.0001)$. There were no effects or interactions in relation to Experience $(F \mathrm{~s}<1, p \mathrm{~s}>.49)$. To explore the PRI Type x Response interaction, we conducted a series of paired $t$-tests. These revealed significant differences between all levels of both factors $(t \mathrm{~s}>6, p \mathrm{~s}<.0001)$, apart from between the halt-patrol and continue-patrol responses for indirect PRIs, though this difference approached 
significance $(t(41)=2.6, p=.07)$. Generally speaking, participants spent a longer proportion of trial time fixating PRIs prior to a 'continue-patrol' versus a 'halt-patrol' response, and a greater proportion of trial time fixating direct PRIs versus indirect PRIs.

The analyses, thus far, are very clear. The basic characteristics of the experienced and inexperienced participants' eye movements were very similar, and both participant groups spent a similar proportion of the trial time inspecting the PRIs as they evaluated threat. Also, the increased proportion of trial time spent fixating direct compared to indirect PRIs could reflect a tendency to allocate more attention to direct threat indicators over indirect threat indicators, though recall that this effect may also be due to the increased number and more central location of direct relative to indirect PRIs in the stimulus set.

\section{Table 3}

Table of Means for Proportion of Trial Time spent fixating each PRI Type. Parentheses indicate SEM

\begin{tabular}{cccc}
\hline \multirow{2}{*}{ Experience } & PRI Type & Response & $\begin{array}{c}\text { Mean Proportion of Trial } \\
\text { Time }\end{array}$ \\
& & & \\
\hline \multirow{2}{*}{ Experienced } & Direct & Continue-patrol & $0.71(0.01)$ \\
& & Halt-patrol & $0.79(0.01)$ \\
& Indirect & Continue-patrol & $0.35(0.01)$ \\
& & Halt-patrol & $0.32(0.02)$ \\
& Direct & Continue-patrol & $0.70(0.01)$ \\
& & Halt-patrol & $0.79(0.01)$ \\
& Indirect & Continue-patrol & $0.35(0.02)$ \\
& & Halt-patrol & $0.31(0.02)$ \\
\hline
\end{tabular}


Although we have established that both participant groups spent a similar proportion of each trial fixating PRIs, this does not inform us about the manner in which the PRIs were examined. For example, was the additional time that the inexperienced participants spent fixating the PRIs the result of a single visit to them, or did participants make multiple visits to the PRIs? These questions are important because they could signify that experienced participants were able to evaluate risk based upon fewer visits to the PRIs compared to the inexperienced participants. We therefore compared the number of visits to PRIs, see Table 4. Given the differences in the number and area of the two PRI types, we normalised the number of visits per PRI type by the total area of each PRI type in each display. The ANOVA revealed a significant effect of Experience $(F(1,42)=6.75, p=.013)$, with inexperienced participants making more visits to PRIs than experienced participants. There was also an effect of PRI Type $(F(1,40)=$ $6.52, p=.015)$, with participants tending to revisit direct PRIs more than indirect PRIs, and of Response $(F(1,40)=10.66, p=.002)$, with an increase in visits prior to continue-patrol over haltpatrol responses.

\section{Table 4}

Mean Number of Visits to each Potential Risk Indicator (PRI) Type. Parentheses indicate SEM.

\begin{tabular}{cccr}
\hline \multirow{2}{*}{ Experience } & PRI Type & Response & Mean Number of \\
& & & \\
\hline \multirow{2}{*}{ Experienced } & Direct & Continue-patrol & $8.79(0.52)$ \\
& & Halt-patrol & $7.49(0.48)$ \\
& Indirect & Continue-patrol & $7.79(0.43)$ \\
& & Halt-patrol & $7.15(0.52)$ \\
Inexperienced & Direct & Continue-patrol & $11.18(1.02)$
\end{tabular}




$\begin{array}{ccc} & \text { Halt-patrol } & 10.17(0.82) \\ \text { Indirect } & \text { Continue-patrol } & 10.55(1.27) \\ & \text { Halt-patrol } & 8.66(0.85)\end{array}$

Note. The number of visits has been normalised by the total area of each PRI type in the displays and then multiplied by a constant (1000) to ease interpretation.

Overall, analyses of the PRIs in relation to eye movement behaviour indicate a very clear pattern of results. Both participant groups fixated and examined the two PRI types, furthermore, there was an increase in the visit count for the two PRI types for the inexperienced relative to the experienced participants indicating that the increased response time for inexperienced participants arises as a consequence of increased revisits to PRIs. That is, inexperienced participants are able to recognise those aspects of a scene that represent threat, and then spend time fixating them accordingly. But the inexperienced participants re-inspect PRIs prior to making a decision. Furthermore, as in visual search tasks (Chun \& Wolfe, 1996), the formation of a continue-patrol response requires a more exhaustive examination of the scene (i.e., longer RT, more visits to PRIs, more time fixating, more PRIs visited) than the formation of a haltpatrol response in both experienced and inexperienced participants.

\section{Discussion}

In the present study, we examined how experience influences both information-sampling behaviour and decision-making during a novel risk assessment task. Previous studies in a number of different domains have found that both information-sampling and decision-making are modulated by experience (for a reivew, see Reingold \& Sheridan, 2011). However, some studies have found that experienced observers are both more accurate and more rapid than inexperienced observers (Kundel \& Nodine, 1975; Nodine \& Kundel, 1987; Nodine \& Mello-Thoms, 2000), 
while others have found that experienced observers are more accurate but less rapid than inexperienced observers (Biggs et al., 2013; Biggs \& Mitroff, 2014a, 2014b). We recorded the eye movement behaviour of a group of military personnel as they assessed the presence of threat in a series of scenes, and who were asked to decide whether they would continue a patrol through the scene depicted or halt a patrol through the scene depicted due to the presence of a high level of risk.

We began by examining the time taken for participants to respond in the task. We found that the experienced participants responded much more rapidly than the inexperienced participants, as has been reported previously in studies of radiographic image inspection. Furthermore, we also examined the factors that influenced how likely participants were to respond 'halt-patrol'. We found that the halt-patrol decisions were more likely for images that we knew contained an IED, and were more likely for images that contained a greater area of Direct PRIs. Clearly, this demonstrates that the halt-patrol decisions are made upon a rational basis, in the sense that they are more likely when the primary form of threat is present (IEDs), and are also more likely when PRIs related to IEDs are present within the scenes. Furthermore, these analyses also revealed that the inexperienced participants were more likely to respond 'halt-patrol' overall than the experienced participants. We did find that the size of the Indirect PRIs did not influence decision-making, though this does not mean that Indirect PRIs are not used as part of the decision-making, since participants did spend time inspecting them during the trials. It should also be considered that Indirect PRIs were smaller in size, and had less variability in size, than the Direct PRIs, so there the lack of an effect may also be due to power issues. For the time being, we conclude that Direct PRIs have a clear influence on decision-making, with 'halt-patrol' decisions being more likely as the size of Direct PRIs increasing. 
We examined the participants' eye movement behaviour in order to gain insights into the information-sampling that took place while participants formulated their risk assessment decisions. Although previous studies have found that experience leads participants to become more sensitive to the task-relevant locations within a stimulus (Charness, Reingold, Pomplun, \& Stampe, 2001; Kundel \& Nodine, 1975; Nodine \& Kundel, 1987; Nodine \& Mello-Thoms, 2000; Underwood, Crundall, \& Chapman, 2002), demonstrating a modulation of information-sampling behaviour, here we found that participants were equally likely to fixate the PRIs, demonstrating that the selectivity of information-sampling was not influenced by experience. Despite this lack of a difference in information-sampling in terms of the regions fixated by the participants, by examining participants' eye movement behaviour, we were able to determine the additional information-sampling that the inexperienced participants engaged in during the additional time that they spent searching. These examinations revealed that, during the additional time spent searching, the inexperienced participants repeatedly visited PRIs. In the reality of theatre, threats of many kinds must be balanced and decisions must be made quickly as delay can lend opportunities to the enemy. Anecdotal evidence based on interviews with personnel that have completed tours of duty in Afghanistan indicate that they believe that they become more familiar with the environment over time and that this supports better and more rapid decision making.

What factors might be driving the differences in both the time required to formulate a decision, and the nature of the decisions made by the inexperienced and experienced participants? These factors could include a development in the ability to 'break' camouflage (X. Chen \& Hegdé, 2012; Hegdé, Fang, Murray, \& Kersten, 2008; Hegdé, Thompson, Brady, \& Kersten, 2012), as well as experience with learning the important and 'informative' features to focus on (Blair, Watson, \& Meier, 2009; Blair, Watson, Walshe, \& Maj, 2009; L. Chen, Meier, 
Blair, Watson, \& Wood, 2013). One further possibility stands out: the role of expectations regarding the likelihood of threat being present (Godwin, Menneer, Cave, \& Donnelly, 2010; Godwin, Menneer, Cave, Helman, et al., 2010; Menneer, Donnelly, Godwin, \& Cave, 2010; Van Wert, Horowitz, \& Wolfe, 2009; Wolfe \& Van Wert, 2010; Wolfe et al., 2007; Wolfe, Horowitz, \& Kenner, 2005). Previous studies examining the prevalence effect in visual search have found that, when targets are presented on a high proportion of trials ( $>50 \%$ of trials), then participants show a tendency to spend longer times searching, as well as a tendency to report that they believe targets to be present. This is very similar to the pattern of effects observed for the inexperienced participants, who spent longer searching and were more likely to respond 'halt-

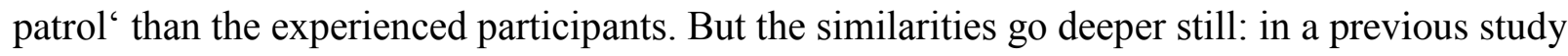
(Godwin et al., 2014), we also found that, when participants expected a target to be present, they made repeated visits to objects, just as the inexperienced participants did here with the PRIs.

One final alternative is that the experienced and novice participants may have been engaged in the task under the assumption that different reward and punishment structures were in place or because of differing levels of anxiety. When participants are penalised for failing to detect a target, then they are more likely to respond that the target is present, even when it is not (Chun \& Wolfe, 1996; Macmillan \& Creelman, 2005). In a recent study, Cooper et al. (2013) found that CCTV operators were more likely to respond that they had detected dangerous or suspicious behaviour in the video streams that they were monitoring when under conditions of induced anxiety. The experienced participants in our experiment will have had considerable and varying experiences associated with success and failure in risk assessment decisions. In some cases, their decisions may have had significant outcomes (injury or death) for colleagues where threats were not detected. However, experienced participants will also be aware that false alarms 
(i.e., claims that risk is present in the environment when that is, in fact, not correct) will also have significant consequences on time and resources and exposure to secondary risks such as small arms fire. The inexperienced participants, on the other hand, will have had no such experience. It is important to note that, since we did not measure the level of anxiety of our participants in the present study, this final explanation requires further experimentation to fully understand the influence of anxiety upon risk assessment behaviour in scenes of this type.

\section{Summary}

To summarise, our results demonstrate that experience with risk assessment primarily modulates decision-making behaviour. Overall it is clear that experience with conducting risk assessment for the presence of IEDs enables observers to more rapidly reach decisions, drawing upon their extensive prior experience as they do so. When observers lack experience, much more time is required for them to reach a decision, and, furthermore, they are more likely to respond cautiously, reporting that an area or environment is not safe to proceed into. From a training perspective, it seems important to emphasise the many various forms of PRIs to inexperienced participants, as well as to provide them with experience in detecting safe as well as unsafe environments, along with a library of exemplars of scenes that belong in each of these categories. This should enable them to build up a more fine-grained internal representation of the two different types of environment, and, as a result, become more readily able to distinguish threat from non-threat environments and shift their behaviour to be more similar to the experienced participants involved in the present study. 


\section{References}

Baayen, R. H. (2008). Analyzing Linguistic Data A practical introduction to statistics. Cambridge: Cambridge University Press.

Biggs, A. T., Cain, M. S., Clark, K., Darling, E. F., \& Mitroff, S. R. (2013). Assessing visual search performance differences between Transportation Security Administration Officers and nonprofessional visual searchers, (June), 37-41.

Biggs, A. T., \& Mitroff, S. R. (2014a). Differences in multiple-target visual search performance between non-professional and professional searchers due to decision-making criteria. British Journal of Psychology (London, England : 1953), 1-13. doi:10.1111/bjop.12096

Biggs, A. T., \& Mitroff, S. R. (2014b). Different predictors of multiple-target search accuracy between nonprofessional and professional visual searchers. Quarterly Journal of Experimental Psychology (2006), 67(7), 1335-48. doi:10.1080/17470218.2013.859715

Blair, M. R., Watson, M. R., \& Meier, K. M. (2009). Errors, efficiency, and the interplay between attention and category learning. Cognition, 112(2), 330-6.

doi:10.1016/j.cognition.2009.04.008

Blair, M. R., Watson, M. R., Walshe, R. C., \& Maj, F. (2009). Extremely selective attention: eye-tracking studies of the dynamic allocation of attention to stimulus features in categorization. Journal of Experimental Psychology. Learning, Memory, and Cognition, 35(5), 1196-206. doi:10.1037/a0016272

Cain, M. S., Adamo, S. H., \& Mitroff, S. R. (2013). A taxonomy of errors in multiple-target visual search. Visual Cognition, 21(7), 899-921. doi:10.1080/13506285.2013.843627

Chapman, P. R., \& Underwood, G. (1998). Visual search of driving situations: danger and experience. Perception, 27(8), 951-964. doi:10.1068/p270951

Charness, N., Reingold, E. M., Pomplun, M., \& Stampe, D. M. (2001). The perceptual aspect of skilled performance in chess: evidence from eye movements. Memory \& Cognition, 29(8), 1146-52. doi:10.3758/BF03206384

Chen, L., Meier, K. M., Blair, M. R., Watson, M. R., \& Wood, M. J. (2013). Temporal characteristics of overt attentional behavior during category learning. Attention, Perception \& Psychophysics, 75(2), 244-56. doi:10.3758/s13414-012-0395-8

Chen, X., \& Hegdé, J. (2012). Learning to break camouflage by learning the background. Psychological Science, 23(11), 1395-403. doi:10.1177/0956797612445315

Chun, M. M., \& Wolfe, J. M. (1996). Just say no: how are visual searches terminated when there is no target present? Cognitive Psychology, 30(1), 39-78. doi:10.1006/cogp.1996.0002 
Cooper, R., Howard, C. J., Attwood, A. S., Stirland, R., Rostant, V., Renton, L., ... Munafò, M. R. (2013). Acutely induced anxiety increases negative interpretations of events in a closedcircuit television monitoring task. Cognition \& Emotion, 27(2), 273-82. doi:10.1080/02699931.2012.704352

Godwin, H. J., Menneer, T., Cave, K. R., \& Donnelly, N. (2010). Dual-target search for high and low prevalence X-ray threat targets. Visual Cognition, 18(10), 1439-1493. doi:doi:10.1080/13506285.2010.500605

Godwin, H. J., Menneer, T., Cave, K. R., Helman, S., Way, R. L., \& Donnelly, N. (2010). The impact of relative prevalence on dual-target search for threat items from airport X-ray screening. Acta Psychologica, 134(1), 79-84. doi:doi:10.1016/j.actpsy.2009.12.009

Godwin, H. J., Menneer, T., Cave, K. R., Thaibsyah, M., \& Donnelly, N. (2014). The effects of increasing target prevalence on information processing during visual search. Psychonomic Bulletin \& Review. doi:10.3758/s13423-014-0686-2

Godwin, H. J., Menneer, T., Riggs, C. A., Cave, K. R., \& Donnelly, N. (2015). Perceptual Failures in the Selection and Identification of Low-Prevalence Targets in Relative Prevalence Visual Search. Attention, Perception \& Psychophysics, 77(1), 150-9. doi:10.3758/s13414-014-0762-8

Hegdé, J., Fang, F., Murray, S. O., \& Kersten, D. (2008). Preferential responses to occluded objects in the human visual cortex. Journal of Vision, 8(4), 1-16. doi:10.1167/8.4.16

Hegdé, J., Thompson, S. K., Brady, M., \& Kersten, D. (2012). Object recognition in clutter: cortical responses depend on the type of learning. Frontiers in Human Neuroscience, 6(June), 170. doi:10.3389/fnhum.2012.00170

Kundel, H. L., \& Nodine, C. F. (1975). Interpreting chest radiographs without visual search. Radiology, 116, 527-532.

Macmillan, N. A., \& Creelman, C. D. (2005). Detection theory: A user's guide. (2nd ed.). Cambridge: Cambridge University Press.

Menneer, T., Donnelly, N., Godwin, H. J., \& Cave, K. R. (2010). High or low target prevalence increases the dual-target cost in visual search. Journal of Experimental Psychology:Applied, 16(2), 133-144. doi:10.1037/a0019569

Nodine, C. F., \& Kundel, L. (1987). Using eye movements to study visual search and to improve tumor detection. RadioGraphics, 7(6), 1241-1250.

Nodine, C. F., \& Mello-Thoms, C. (2000). The nature of expertise in radiology. In J. Beutel, H. L. Kundel, \& R. L. Van Metter (Eds.), The handbook of medical image: Physics and psychophysics. (pp. 859-894). Bellingham, WA: SPIE Press. 
Reingold, E. M., \& Sheridan, H. (2011). Eye movements and visual expertise in chess and medicine. In S. P. Liversedge, I. D. Gilchrist, \& S. Everling (Eds.), Oxford handbook on eye movements (pp. 523-550). Oxford, UK: Oxford University Press.

Schwark, J. D., MacDonald, J., Sandry, J., \& Dolgov, I. (2013). Prevalence-based decisions undermine visual search. Visual Cognition, 21(5), 541-568. doi:10.1080/13506285.2013.811135

Underwood, G., Crundall, D., \& Chapman, P. (2002). Selective searching while driving: the role of experience in hazard detection and general surveillance. Ergonomics, 45(1), 1-12. doi:10.1080/00140130110110610

Van Wert, M. J., Horowitz, T. S., \& Wolfe, J. M. (2009). Even in correctable search, some types of rare targets are frequently missed. Attention, Perception \& Psychophysics, 71, 541-553. doi:10.3758/APP.71.3.541

Wolfe, J. M., Brunelli, D. N., Rubinstein, J., \& Horowitz, T. S. (2013). Prevalence effects in newly trained airport checkpoint screeners : Trained observers miss rare targets , too, 13(3), 1-9. doi:10.1167/13.3.33.doi

Wolfe, J. M., Horowitz, T. S., \& Kenner, N. M. (2005). Rare items often missed in visual searches. Nature, 435(May), 6-7. doi:10.1038/435439a

Wolfe, J. M., Horowitz, T. S., Van Wert, M. J., Kenner, N. M., Place, S. S., \& Kibbi, N. (2007). Low target prevalence is a stubborn source of errors in visual search tasks. Journal of Experimental Psychology: General, 136(4), 623-38. doi:10.1037/0096-3445.136.4.623

Wolfe, J. M., \& Van Wert, M. J. (2010). Varying target prevalence reveals two dissociable decision criteria in visual search. Current Biology, 20(2), 121-4. doi:10.1016/j.cub.2009.11.066 